\title{
Exercício aeróbio e pressão arterial no idoso
}

\author{
ROMEU MENDES,* J. L. THEMUDO BARATA**
}

\begin{abstract}
Resumo
Introdução: Vários organismos internacionais têm recomendado o exercício aeróbio como medida de prevenção, tratamento e controlo da hipertensão arterial na população em geral. No entanto, o efeito do exercício aeróbio na pressão arterial da população idosa ainda é uma área pouco estudada e os resultados dos estudos nem sempre são consistentes.

Objectivo: Este estudo de revisão tem como objectivo analisar a influência crónica e aguda do exercício aeróbio nos valores da pressão arterial da populacão idosa.

Metodologia: Foi realizada uma pesquisa nas bases de dados Pubmed e Highwire Press. Foram também pesquisadas as recomendações de várias organizações internacionais na área da cardiologia. Incluíram-se no estudo 67 artigos.

Conclusões: 0 exercício aeróbio, quer de forma aguda ou crónica, parece diminuir tanto os niveis de pressão arterial clínica, como de pressão arterial ambulatória, dos sujeitos idosos, especialmente dos hipertensos. Parece assim existirem evidências para considerar o exercício aeróbio como estratégia terapêutica não farmacológica e de modificação do estilo de vida, essencial para a prevenção, tratamento e controlo da hipertensão nos indivíduos idosos. Os profissionais de saúde devem ter um papel activo no aconselhamento de exercício à população idosa, contribuindo assim para a prevenção e controlo das doenças cardiovasculares.
\end{abstract}

Palavras-Chave: Exercício; Pressão Arterial; Idoso.

*Fisiologista do Exercício, Mestre em Actividade Fisica para a $3^{a}$ Idade **Médico Especialista em Medicina Interna e Medicina Desportiva, Doutorado em Medicina, Professor da Faculdade de Ciências da Saúde da Universidade da Beira Interior, Director do Serviço de Nutrição e Actividade Fisica do Centro Hospitalar Cova da Beira

\section{INTRODUÇ̃̃O}

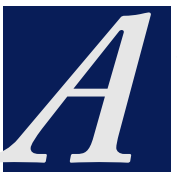

$\mathrm{s}$ fortes evidências dos beneficios cardiovasculares da actividade fisica e do exercício regular têm levado organizações como a World Health Organization e a International Society of Hypertension, ${ }^{1}$ a European Society of Hypertension e a European Society of Cardiology, ${ }^{2}$ a British Hypertension Society, ${ }^{3}$ a American Heart Association, ${ }^{4}$ e o American College of Sports Medicine, ${ }^{5}$ a recomendarem a actividade física de uma forma geral e o exercício aeróbio em particular, como uma estratégia de intervenção não farmacológica e de modificação do estilo de vida da população, fundamental na prevenção primária, tratamento e controlo da hipertensão arterial e na prevenção das doenças cardiovas- culares (DCV). Apesar de existirem claras evidências de que o exercício aeróbio tem repercussões crónicas e agudas na diminuição da pressão arterial (PA) de sujeitos jovens e adultos, o seu efeito nos indivíduos idosos ainda é uma área pouco estudada e os resultados dos estudos nem sempre são consistentes. Este estudo de revisão tem como objectivo analisar a influência crónica e aguda do exercício aeróbio nos valores da PA da população idosa, assim como os mecanismos da hipotensão pós-exercício e o papel do exercício aeróbio no controlo da hipertensão arterial.

\section{METODOLOGIA}

Foi realizada uma pesquisa nas bases de dados Pubmed e Highwire Press, de artigos até Setembro de 
2007, com especial enfoque nos últimos 5 anos. Foram utilizadas as seguintes palavras-chave: Exercício; Pressão Arterial; Idoso (Exercise; Blood Pressure; Aged). Foram seleccionados todos os artigos cujo título ou resumo foi considerado relevante no contexto da revisão em causa. Posteriormente procedeu-se à obtenção dos artigos em texto integral. Foram ainda pesquisadas as recomendações de várias organizações como a World Health Organization, ${ }^{1}$ a International Society of Hypertension, ${ }^{1}$ a European Society of Cardiology, ${ }^{2}$ a European Society of Hypertension, ${ }^{2}$ a British Hypertension Society, ${ }^{3}$ a American Heart Association ${ }^{4}$ o o American College of Sports Medicine. ${ }^{5}$ Foram incluídos neste estudo 67 artigos.

\section{EFEITOS dO EXERCícIO AeróbIO na Pressão ARterial}

Um dos efeitos da actividade fisica e do exercício parece ser a diminuição da PA. ${ }^{6-8}$ A PA parece baixar após uma sessão isolada de exercício, após um programa de exercício regular ou, simplesmente, como consequência do aumento da actividade física habitual. ${ }^{5.9,10}$ Apesar de, em geral, ser aceite que a actividade fisica e o exercício reduzem a PA, são ainda insuficientes os estudos em diferentes populações ou com protocolos de exercício diferentes. No entanto, parece ser consensual que o exercício aeróbio é o tipo de exercício mais eficaz para diminuir os valores de PA. $2,5,11,12$ Os exercícios resistidos, ${ }^{\dagger}$ utilizados para o desenvolvimento da resistência muscular, são também recomendados, embora a sua eficácia na redução da PA

$\dagger$ Exercícios realizados com o uso de resistências à contracção muscular (máquinas de musculação, pesos livres, bandas elásticas, peso corporal, etc). seja menor do que a obtida pelo exercício aeróbio generalizado e, por isso, devem ser usados como complemento do mesmo..$^{5,9,13,14}$

\section{Efeitos crónicos}

Vários estudos demonstraram que um programa de exercício aeróbio regular é eficaz para induzir a diminuição da PA de repouso em sujeitos jovens e adultos normoten$\operatorname{sos}^{15-18}$ e hipertensos, ${ }^{15-22}$ de ambos os sexos. Contudo, a diminuição da PA associada à prática regular de exercício aeróbio parece ser mais acentuada em individuos hipertensos do que em normotensos. ${ }^{5,9,17,18} \mathrm{O}$ exercício aeróbio regular parece, também, reduzir a pressão arterial ambulatória (PAA) em sujeitos normotensos ${ }^{23}$ e hipertensos. ${ }^{23-25}$ Estudos de meta-análise revelaram que as reduções na PA parece serem independentes da frequência semanal, da duração e da intensidade das sessões de exercício aeróbio. ${ }^{16,18}$ Um estudo realizado por Elley et al., ${ }^{26}$ demonstrou que quatro sessões de 10 minutos de marcha rápida por dia foram tão efectivas como 40 minutos de marcha rápida contínua, na redução da pressão arterial de indivíduos hipertensos. Para além de diminuir os valores de PA, o exercício aeróbio regular revelou-se eficaz na redução da hipertrofia ventricular esquerda, em individuos com hipertensão severa, ${ }^{19} \mathrm{e}$ na melhoria da vasodilatação dependente do endotélio vascular, através do aumento da libertação de óxido nítrico, em indivíduos normotensos e hipertensos. ${ }^{15}$

\section{Efeitos crónicos no idoso}

Vários estudos, em idosos hipertensos, demonstraram que o exercício aeróbio regular também parece reduzir a PA clínica, ${ }^{27-32}$ a $P A A^{31}$ e os valores da PA medidos em casa. ${ }^{33} \mathrm{Em}$ idosos normotensos, Maeda et al. ${ }^{34}$ verificaram que o exercício aeróbio regular foi eficaz na redução da PA de repouso, e o estudo de Jessup et al. ${ }^{35}$ demonstrou que um programa de exercício aeróbio regular foi igualmente eficaz na redução da PAA. Contudo, os estudos de Dengel et al., ${ }^{32}$ Tanaka et al. ${ }^{36}$ e DeSouza et al. ${ }^{37}$ não revelaram reduções significativas da PA de repouso de idosos normotensos, após um programa de exercício aeróbio regular. Por sua vez, um estudo de meta-análise realizado por Kelley \& Kelley $^{38}$ apenas encontrou pequenas reduções na PAS, quer em idosos normotensos, quer em hipertensos. As reduções na PA verificadas nos idosos parece serem inferiores às observadas nas faixas etárias mais baixas ${ }^{27}$ e parece serem mais acentuadas nos indivíduos hipertensos do que nos normotensos. ${ }^{5,9,32,38}$ Nesta população, o exercício aeróbio regular demonstrou também estar associado à diminuição da espessura da parede ventricular esquerda de sujeitos hipertensos; ${ }^{28}$ à atenuação da diminuição da complacência arterial associada ao envelhecimento, e até a sua recuperação parcial, em sujeitos sedentários e normotensos; ${ }^{36}$ ao aumento da vasodilatação mediada pelo endotélio, em sujeitos normotensos e sedentários; ${ }^{37}$ e ao aumento da produção de óxido nítrico em indivíduos normotensos sedentários. $^{34}$

\section{Efeitos agudos}

A hipotensão pós-exercício (HPE) é um fenómeno agudo de diminuição dos valores da PA de repouso, num período de tempo subsequente (minutos/horas) à realização de uma sessão de exercício. ${ }^{39} \mathrm{~A}$ HPE parece ocorrer em indivíduos de género e idades diferentes, em normoten$\operatorname{sos}^{40-44}$ e hipertensos, ${ }^{40,45}$ independentemente da intensidade,,${ }^{9,41,43} \mathrm{du}$ - 
ração, ${ }^{39-40}$ modo de exercício ${ }^{39}$ e da quantidade de massa muscular activa. ${ }^{45}$ Todavia, os resultados de alguns estudos sugerem que a magnitude e duração da HPE parecem ser dependentes da intensidade ${ }^{44} \mathrm{e}$ duração ${ }^{42}$ da sessão de exercício. Outros autores referem ainda que, apesar de se poder detectar o efeito de HPE em individuos normotensos, este é muito menos frequente e de menor magnitude, em comparação com o que se regista nos individuos hipertensos. ${ }^{9,39,46}$ Nos estudos anteriormente referidos, a HPE foi observada em condições altamente controladas (ambiente de laboratório), com os sujeitos em repouso logo após a realização da sessão de exercício e durante um período limitado de tempo (entre 60 a 90 minutos). Desta forma, as medidas da PA, registadas após exercício e em repouso, podem não corresponder aos valores reais da PA que se verificariam se os individuos estivessem envolvidos nas tarefas da vida diária. ${ }^{47} \mathrm{~A}$ HPE apenas tem utilidade clínica se tiver uma duração prolongada durante o período de tempo em que ocorrem as actividades do dia-a-dia. ${ }^{39}$ A utilização da monitorização ambulatória da pressão arterial (MAPA) permite avaliar a PA e, eventualmente, determinar o potencial efeito de HPE sem que as rotinas do dia-a-dia sejam modificadas. ${ }^{9,48}$ Contudo, os estudos que analisaram a HPE através da MAPA apresentam resultados inconsistentes, talvez devido às diferentes metodologias utilizadas no que diz respeito ao tipo de amostra e ao protocolo de exercício. Uma sessão isolada de exercício aeróbio parece diminuir significativamente, e em comparação com uma sessão de controlo, a PAA de indivíduos hipertensos ${ }^{23,49-53}$ e de indivíduos normotensos. ${ }^{23,54,55}$ No entanto, a maioria dos estudos realizados em sujeitos normotensos não apontam nesse sentido. ${ }^{44,47,50-52}$ A variável que parece provocar maior inconsistência nos resultados é o nível da PA inicial dos indivíduos da amostra. $\mathrm{O}$ efeito HPE não parece ser tão consistente nos individuos normotensos como nos sujeitos hipertensos, e as reduções da PA, após o exercício, parece serem maiores nos sujeitos que apresentam valores de PA mais elevados antes do exercício..$^{23,39,47,49,54}$ A ausência de efeito hipotensivo, após o exercício nos sujeitos normotensos, pode dever-se aos baixos valores iniciais (antes do exercício) da PA. ${ }^{44,47}$ Segundo alguns estudos, a PA antes do exercício e a PA clínica dos indivíduos são das variáveis com mais influência na magnitude e na duração da HPE, especialmente observada através da MAPA. 5,23,39,49,54,55

\section{Efeitos agudos no idoso}

São ainda poucos os estudos que analisaram o efeito agudo do exercício aeróbio na PA de sujeitos idosos. Hagberg et al. ${ }^{56}$ desenvolveram um estudo para determinar a eventual ocorrência de um efeito hipotensivo pós-exercício em sujeitos idosos hipertensos. A amostra foi submetida a duas sessões de exercício aeróbio com diferentes intensidades (50 e $70 \%$ do volume máximo de oxigénio). Os resultados revelaram que o exercício às duas intensidades, consideradas no estudo, induziu uma diminuição significativa (comparativamente a uma sessão de controlo) da PAS medida em repouso, durante um período de 3 horas, e em condições de laboratório. Um estudo realizado por Taylor-Tolbert et al..$^{57}$ verificou que uma sessão de exercício aeróbio, constituída por 3 fracções de 15 minutos de marcha em tapete ergómetro, a uma intensidade de $70 \%$ do volume máximo de oxigénio ( $\mathrm{VO}_{2} \max$ ), reduziu significativamente (quando comparada com uma sessão de controlo) a PAA de um grupo de sujeitos obesos, hipertensos, de meia idade e idosos. A pressão arterial sistólica (PAS) foi reduzida entre 6 a $13 \mathrm{mmHg}$ nas 16 horas após o exercício, e a pressão arterial diastólica (PAD) esteve reduzida durante 12 das primeiras 16 horas após o exercício. A média da PAS e PAD das 24 horas também diminuiu significativamente em relação à sessão de controlo, assim como a PAS e PAD diurna e nocturna. Brandão et al. ${ }^{58}$ verificaram que uma sessão de exercício aeróbio realizado em ciclo-ergómetro, a uma intensidade de $50 \%$ do $\mathrm{VO}_{2} \max$, com a duração de 45 minutos, diminui significativamente a PAA em idosos hipertensos, mas não em idosos normotensos, durante uma MAPA de 22 horas, relativamente a uma sessão de controlo. Apesar de parecer haver evidências de que a HPE também ocorre nos sujeitos idosos, este fenómeno parece ocorrer apenas nos indivíduos hipertensos, quer na PA medida em repouso e em condições de laboratório, ${ }^{56}$ quer na PAA.${ }^{57-58}$ Porém, sendo o número de estudos já realizados relativamente escasso, outros mais são necessários para confirmar o potencial efeito agudo do exercício aeróbio na PA de indivíduos idosos, especialmente nos sujeitos normotensos. ${ }^{5,38}$

\section{MECANISMOS DA Hipotensão Pós-Exercício}

O mecanismo exacto da HPE ainda é desconhecido, mas é provavelmente uma interacção de vários factores. ${ }^{5,9,13,39}$ A PA é determinada pelo débito cardíaco e pelas resistências vasculares periféricas..$^{5,39,46}$ A HPE pode ser vista como um estado he- 
modinâmico de transição entre o estado de exercício dinâmico, envolvendo grandes massas musculares, e o estado de repouso. ${ }^{46} \mathrm{O}$ exercício aeróbio dinâmico afecta, profundamente, o sistema cardiovascular. As resistências vasculares periféricas são reduzidas durante o exercício devido a uma vasodilatação predominante nos músculos exercitados. O débito cardíaco aumenta e tem, como resultado, um ligeiro aumento da PAD e um acréscimo mais pronunciado da PAS. Quando o exercício cessa, o débito cardíaco diminui rapidamente, enquanto as resistências vasculares periféricas recuperam lentamente. Este desequilíbrio nas duas determinantes da PA resulta numa PA menor do que antes do exercício. ${ }^{5,9,14,39,46,48}$ A HPE parece, assim, ocorrer devido à diminuição das resistências vasculares periféricas, provocada por uma vasodilatação predominante nos músculos exercitados, e não tanto às alterações do débito cardíaco. ${ }^{5,39,44,46} \mathrm{~A}$ vasodilatação parece estar associada a duas alterações na regulação vascular simpática: a componente vascular e a componente neural. A componente neural associada à vasodilatação resulta da diminuição do fluxo da actividade do nervo simpático na rede vascular do músculo-esquelético. Em condições de repouso, a actividade nervosa simpática no músculo está sob forte regulação dos baroreflexos arteriais. Durante o exercício, os baroreflexos são ajustados para um nivel superior e a actividade simpática é aumentada. Após o exercício, estes reflexos são reajustados para baixas pressões e o fluxo simpático do sistema nervoso central torna-se mais baixo do que os valores anteriores ao exercício. ${ }^{5,13,39,46,48}$ A componente vascular refere-se à atenuação da resposta vascular à vasoconstrição simpática, assim como a influência potencial das substâncias vasodilatadoras locais e circulantes. Para além da redução do fluxo simpático, a sensibilidade vascular à estimulação dos receptores $\alpha$-adrenérgicos é comprometida de tal forma que a resistência vascular é reduzida para um determinado nivel de actividade nervosa após o exercício. Vários factores associados ao exercício, como o aumento do fluxo sanguíneo e das concentrações de catecolaminas, estimulam a libertação de óxido nítrico, de prostaglandinas, de adenosina e de adenosina trifosfato a partir do endotélio vascular. Estas substâncias atenuam a resposta vasoconstritora à estimulação dos receptores $\alpha$-adrenérgicos e facilitam a vasodilatação. ${ }^{5,13,39,46,59}$ No entanto, em idosos hipertensos, a HPE poderá ocorrer devido a outros mecanismos. Hagberg et al. ${ }^{56}$ observaram uma redução do débito cardíaco devido a uma diminuição do volume sistólico, após uma sessão aguda de exercício aeróbio, e um aumento das resistências vasculares periféricas, em indivíduos idosos hipertensos. Os autores sugerem que outros mecanismos, como o retorno venoso e a contractilidade do miocárdio, poderão estar envolvidos na HPE. Brandão et al. ${ }^{58}$ observaram, também, uma diminuição do débito cardíaco devido a uma redução do volume sistólico e a uma diminuição do volume diastólico final, após uma sessão aguda de exercício aeróbio em idosos com hipertensão. A resposta da PA após exercício pode, também, estar associada a determinismos genéticos. ${ }^{5,9,57,60,61}$ Mais investigações são necessárias para determinar os mecanismos responsáveis pela HPE, quer em indivíduos adultos, quer em indivíduos idosos. ${ }^{9,39} \mathrm{E}$ importante perceber o fenómeno da HPE para tentar explicar a diminu- ição da PA, induzida pelo exercício crónico. ${ }^{49,51}$ Uma sessão de exercício isolado produz uma resposta cardiovascular e metabólica aguda. A repetição frequente destas sessões isoladas produz uma adaptação persistente, referida como adaptação crónica ao exercício. ${ }^{62}$ As adaptações observadas em resposta ao exercício regular poderão resultar do efeito cumulativo dos efeitos agudos sucessivos ao longo dos programas de exercício. ${ }^{5,46,52,57,62}$ No entanto, esta premissa não parece estar ainda suficientemente estudada. ${ }^{46}$

\section{EXERCICIO AERÓBIO e HIPERTENSÃo ARTERIAL}

Considerando que o exercício aeróbio isolado tem um efeito hipotensivo e que a sua realização, de forma regular, encerra um potencial de longo prazo na redução da PA, então, poderá afirmar-se que o exercício aeróbio se constitui como uma medida de modificação do estilo de vida, com elevado valor para a prevenção primária da hipertensão, e como estratégia terapêutica não farmacológica importante para o tratamento e controlo da mesma. ${ }^{2,3,5,9,63-65}$ De uma forma geral, é recomendada uma frequência de exercício diária ou quase diária, com um mínimo de 30 minutos de duração, consecutivos ou acumulados ao longo do dia (em períodos mínimos de 10 minutos), de exercício do tipo aeróbio, como caminhar de forma rápida, a uma intensidade moderada de, por exemplo, 40 a $60 \%$ do volume de oxigénio de reserva ou da frequência cardíaca de reserva. ${ }^{2,3,5,26,65-67}$

\section{CONCLUSÕES}

O exercício aeróbio, quer de forma 
aguda ou crónica, parece diminuir tanto os níveis de PA clínica, como de PAA, dos sujeitos idosos, especialmente dos hipertensos. Parece existirem evidências para considerar o exercício aeróbio como estratégia terapêutica não farmacológica e de modificação do estilo de vida, essencial para a prevenção, tratamento e controlo da hipertensão nos indivíduos idosos. Os profissionais de saúde devem ter um papel activo no aconselhamento de exercício à população idosa, contribuindo assim para a prevenção e controlo das DCV.

\section{REFERÊNCIAS BIBLIOGRÁFICAS}

1. Whitworth JA. 2003 World Health Organization (WHO) / International Society of Hypertension (ISH) statement on management of hypertension. J Hypertens 2003 Nov; 21 (11): 1983-92.

2. European Society of Hypertension European Society of Cardiology Guidelines Committee. 2003 European Society of Hypertension - European Society of Cardiology guidelines for the management of arterial hypertension. J Hypertens 2003 Jun; 21 (6): 1011-53.

3. Williams B, Poulter NR, Brown MJ, Davis M, McInnes GT, Potter JF, et al. Guidelines for management of hypertension: report of the Fourth Working Party of the British Hypertension Society, 2004-BHS IV. J Hum Hypertens 2004 Mar; 18 (3): 139-85.

4. Williams MA, Fleg JL, Ades PA, Chaitman BR, Miller NH, Mohiuddin SM, et al. Secondary prevention of coronary heart disease in the elderly (with emphasis on patients with 75 or more years of age). An American Heart Association Scientific Statement from the Council on Clinical Cardiology Subcommittee on Exercise, Cardiac Rehabilitation and Prevention. Circulation 2002 Apr 9; 105 (14): 1735-43.

5. Pescatello LS, Franklin BA, Fagard R, Farquhar WB, Kelley GA, Ray CA. Exercise and hypertension. American College of Sports Medicine Position Stand. Med Sci Sports Exerc 2004 Mar; 36 (3): 533-53.
6. Shephard RJ, Balady GJ. Exercise as cardiovascular therapy. Circulation 1999 Feb 23; 99 (7): 963-72.

7. Nied RJ, Franklin B. Promoting and prescribing exercise for the elderly. Am Fam Physician 2002 Feb 1; 65 (3): 419-26.

8. Lopes H, Barreto-Filho J, Riccio G. Tratamento não-medicamentoso da hipertensão. Rev Soc Cardiol Estado São Paulo 2003 jan-fev; 13 (1): 148-55.

9. Wallace J. Exercise in hypertension: a clinical review. Sports Med 2003; 33 (8): 585-98.

10. Auer J, Berent R, Prenninger M, Weber T, Kritzinger K, Veits M, et al. Shortterm effects of a single exercise bout at moderate altitude on blood pressure. J Sport Rehabil 2004 Feb; 13 (1): 19-30.

11. Mazzeo RS, Cavanagh P, Evans WJ, Fiatarone M, Hagberg J, McAudey E, et al. Exercise and physical activity for older adults. American College of Sports Medicine Position Stand. Med Sci Sports Exerc 1998 Jun; 30 (6): 992-1008.

12. Manolis A. Exercise and hypertension. European Society of Hypertension Scientific Newsletter. 2005; 6 (23): 1-2.

13. Baster T, Baster-Brooks C. Exercise and hypertension. Aust Fam Physician 2005 Jun; 34 (6): 419-24.

14. Fagard RH. Exercise is good for your blood pressure: effects of endurance training and resistance training. Clin Exp Pharmacol Physiol 2006 Sep; 33(9): 853-6.

15. Higashi Y, Sasaki S, Kurisu S, Yoshimizu A, Sasaki N, Matsuura H, el al. Regular aerobic exercise augments endothelium-dependent vascular relaxation in normotensive as well as hypertensive subjects: role of endothelium-derived nitric oxide. Circulation 1999 Sep 14; 100: 1194-202.

16. Halbert JA, Silagy CA, Finucane P, Withers RT, Hamdorf PA. The effectiveness of exercise training in lowering blood pressure: a meta-analysis of randomised controlled trials of 4 weeks or longer. J Hum Hypertens 1997 Oct; 11 (10): 641-9.

17. Whelton SP, Chin A, Xin X, He J. Effect of aerobic exercise on blood pressure: a meta-analysis of randomized controlled trials. Ann Intern Med 2002 Apr 2; 136 (7): 493-503.

18. Fagard RH. Exercise characteristics and the blood pressure response to dynamic physical training. Med Sci Sports Exerc 2001 Jun; 33 (6 Suppl.):S484-92.

19. Kokkinos PF, Narayan P, Colleran
JA, Pittaras A, Notargiacomo A, Reda D, et al. Effects of regular exercise on blood pressure and left ventricular hypertrophy in African-American men with severe hipertension. N Engl J Med 1995 Nov 30; 333 (22): 1462-7.

20. Tsai JC, Chang WY, Kao CC, Lu MS, Chen YJ, Chan P. Beneficial effect on blood pressure and lipid profile by programmed exercise training in Taiwanese patients with mild hypertension. Clin Exp Hypertens 2002 May; 24 (4): 315-24.

21. Ishikawa-Takata K, Ohta T, Tanaka $\mathrm{H}$. How much exercise is required to reduce blood pressure in essential hypertensives: a dose-response study. Am J Hypertens 2003 Aug; 16 (8): 629-33.

22. Farinatti $P$ de $T$, Oliveira RB, Pinto VL, Monteiro WD, Francischetti E. Programa domiciliar de exercícios: efeitos de curto prazo sobre a aptidão física e pressão arterial de indivíduos hipertensos. Arq Bras Cardiol 2005 Jun; 84 (6): 473-9.

23. Pescatello LS, Kulikowich JM. The aftereffects of dynamic exercise on ambulatory blood pressure. Med Sci Sports Exerc 2001 Nov; 33 (11): 1855-61.

24. Nami R, Mondillo S, Agricola E, Lenti S, Ferro G, Nami N, et al. Aerobic exercise training fails to reduce blood pressure in nondipper-type hypertension. Am J Hypertens 2000 jun; 13 (6 Pt 1): 593-600.

25. Pescatello LS, Fargo AE, Leach CN Jr, Scherzer HH. Short-term effect of dynamic exercise on arterial blood pressure. Circulation 1991 May; 83 (5): 1557-61.

26. Elley R, Bagrie E, Arroll B. Do snacks of exercise lower blood pressure? A randomised crossover trial. N Z Med J 2006 Jun 2; 119 (1235): U1996.

27. Ishikawa K, Ohta T, Zhang J, Hashimoto S, Tanaka $H$. Influence of age and gender on exercise training-induced blood pressure reduction in systemic hypertension. Am J Cardiol 1999 Jul 15; 84 (2): 192-6.

28. Turner MJ, Spina RJ, Kohrt WM, Ehsani AA. Effect of endurance exercise training on left ventricular size and remodeling in older adults with hypertension. J Gerontol A Biol Sci Med Sci 2000 Apr; 55 (4): M245-51.

29. Stewart KJ, Bacher AC, Turner KL, Fleg JL, Hees PS, Shapiro EP, et al. Effect of exercise on blood pressure in older persons: a randomized controlled trial. Arch Intern Med 2005 Apr 11; 165 (7): 756-62. 
30. Vaitkevicius PV, Ebersold C, Shah MS, Gill NS, Katz RL, Narrett MJ, et al. Effects of aerobic exercise training in community-based subjects aged 80 and older: a pilot study. J Am Geriatr Soc 2002 Dec; 50 (12): 2009-13.

31. Seals DR, Reiling MJ. Effect of regular exercise on 24-hour arterial blood pressure in older hypertensive humans. Hypertension 1991 Nov; 18 (5): 583-92.

32. Dengel DR, Galecki AT, Hagberg JM, Pratley RE. The independent and combined effects of weight loss and aerobic exercise on blood pressure and oral glucose tolerance in older men. Am J Hypertens 1998 Dec; 11 (12): 1405-12.

33. Ohkubo T, Hozawa A, Nagatomi R, Fujita K, Sauvaget C, Watanabe Y, et al. Effects of exercise training on home blood pressure values in older adults: a randomized controlled trial. J Hypertens 2001 Jun; 19 (6): 1045-52.

34. Maeda S, Tanabe T, Otsuki T, Sugawara J, Iemitsu M, Miyauchi T, et al. Moderate regular exercise increases basal production of nitric oxide in elderly women. Hyperten Res 2004 Dec; 27 (12): 947-53.

35. Jessup JV, Lowenthal DT, Pollock ML, turner T. The effects on endurance exercise training on ambulatory blood pressure in normotensive older adults. Geriatr Nephrol Urol 1998; 8 (2): 103-9.

36. Tanaka H, Dinenno FA, Monahan $\mathrm{KD}$, Clevenger CM, DeSouza CA, Seals DR. Aging, habitual exercise, and dynamic arterial compliance. Circulation 2000 Sep 12 ; 102 (11): 1270-5.

37. DeSouza CA, Shapiro LF, Clevenger CM, Dinenno FA, Monahan KD, Tanaka H, et al. Regular aerobic exercise prevents and restores age-related declines in endothelium-dependent vasodilation in healthy men. Circulation 2000 Sep 19; 102 (12): 1351-7.

38. Kelley GA, Sharpe Kelley K. Aerobic exercise and resting blood pressure in older adults: a meta-analytic review of randomized controlled trials. J Gerontol A Biol Sci Med Sci 2001 May; 56 (5): M298-303.

39. MacDonald JR. Potential causes, mechanisms, and implications of post exercise hypotension. J Hum Hypertens 2002 Apr; 16 (4): 225-36.

40. MacDonald JR, MacDougall JD, Hogben CD. The effects of exercise duration on post-exercise hypotension. J Hum Hypertens 2000 Feb; 14 (2): 125-9.
41. MacDonald J, MacDougall J, Hogben $\mathrm{C}$. The effects of exercise intensity on post-exercise hypotension. J Hum Hypertens 1999 Aug; 13 (8): 527-31.

42. Forjaz CL, Santaella DF, Rezende LO, Barreto AC, Negrão CE. A duração do exercício determina a magnitude e a duração da hipotensão pós-exercício. Arq Bras Cardiol 1998 Feb; 70 (2): 99-104.

43. Forjaz CL, Matsudaira Y, Rodrigues FB, Nunes N, Negrão CE. Post-exercise changes in blood pressure, heart rate and rate pressure product at different exercise intensities in normotensive humans. Braz J Med Biol Res 1998 Oct; 31 (10): 1247-55.

44. Forjaz CL, Cardoso CG Jr, Rezk CC, Santaella DF, Tinucci T. Postexercise hypotension and hemodynamics: the role of exercise intensity. J Sports Med Phys Fitness 2004 Mar; 44 (1): 54-62.

45. MacDonald JR, MacDougall JD, Hogben CD. The effects of exercising muscle mass on post exercise hipotension. J Hum Hypertens 2000 May; 14 (5): 317-20.

46. Halliwill JR. Mechanisms and clinical implications of post-exercise hypotension in humans. Exerc Sports Sci Rev 2001 Apr; 29 (2): 65-70.

47. Fisher MM. The effect of aerobic exercise on recovery ambulatory blood pressure in normotensive men and women. Res Q Exerc Sport 2001 Sep;72(3): 267-72.

48. Fagard R. Exercise and hypertension. J Hum Hypertens 1999 Jun; 13 (6): 359-60.

49. Pescatello LS, Guidry MA, Blanchard BE, Kerr A, Taylor AL, Johnson AN, et al. Exercise intensity alters postexercise hypotension. J Hypertens 2004 Oct; 22 (10): 1881-8.

50 . Wallace JP, Bogle PG, King BA, Krasnoff JB, Jastremski CA. The magnitude and duration of ambulatory blood pressure reduction following acute exercise. $\mathrm{J}$ Hum Hypertension 1999 Jun; 13(6): 361-6.

51. Quinn TJ. Twenty-four hour, ambulatory blood pressure responses following acute exercise: impact of exercise intensity. J Hum Hypertens 2000 Sep; 14 (9):547-53.

52. Brownley KA, West SG, Hinderliter AL, Light KC. Acute aerobic exercise reduces ambulatory blood pressure in borderline hypertensive men and women. Am J Hypertens 1996 Mar; 9 (3): 200-6.

53. Pinto A, Raimondo D, Tuttolomondo A, Fernandez P, Arnao V, Licata G. Twenty-four hour ambulatory blood pres- sure monitoring to evaluate effects on blood pressure of physical activity in hypertensive patients. Clin J Sport Med 2006 May; 16 (3): 238-43.

54. Forjaz CL, Tinucci T, Ortega KC, Santaella DF, Mion d Jr, Negrão CE. Factors affecting post-exercise hypotension in normotensive and hypertensive humans. Blood Press Monit 2000 Oct-Dec; 5 (5-6-): 155-62.

55. Bermudes AM, Vassallo DV, Vasquez EC, Lima EG. Monitorização ambulatorial da pressão arterial em indivíduos normotensos submetidos a duas sessões únicas de exercícios: resistido e aeróbio. Arq Bras Cardiol 2004 Jan; 82 (1): 57-64.

56. Hagberg JM, Montain SJ, Martin WH $3^{\text {rd }}$. Blood pressure and hemodynamic responses after exercise in older hypertensive. J Appl Physiol 1987 Jul; 63(1):270-6.

57. Taylor-Tolbert NS, Dengel DR, Brown MD, McCole SD, Pratley RE, Ferrell $\mathrm{RE}$, et al. Ambulatory blood pressure after acute exercise in older men with essential hypertension. Am J Hypertens 2000 Jan; 13 (1): 44-51.

58. Brandão Rondon MU, Alves MJ, Braga AM, Teixeira OT, Barreto AC, Krieger EM, et al. Postexercise blood pressure reduction in elderly hypertensive patients. J Am Coll Cardiol 2002 Feb 20; 39 (4): 676-82.

59. Bacon SL, Sherwood A, Hinderliter A, Blumenthal JA. Effects of exercise, diet and weight loss on high blood pressure. Sports Med 2004; 34 (5): 307-16.

60. Sonna LA, Glueck SB, Jeunemaître $\mathrm{X}$. Exercise, genetics, and blood pressure: focus on "physical exercise and blood pressure with reference to the angiotensinogen M235T polymorphism" and on "angiotensinogen M235T polymorphism associates with exercise hemodynamics in postmenopausal women". Physiol Genomics 2002 Aug 14; 10 (2): 45-7.

61. Choudhury A, Lip GY. Exercise and hypertension. J Hum Hypertens 2005 Aug; 19 (8): 585-7.

62. Thompson PD, Crouse SF, Goodpaster B, Kelley D, Moyna N, Pescatello L. The acute versus the chronic response to exercise. Med Sci Sports Exerc 2001 Jun; 33 (6 Suppl): S438-45.

63. Irigoyen MC, Lacchini S, De Angelis K, Michelini LC. Fisiopatologia da hipertensão: o que avançamos? Rev Soc Cardiol Estado São Paulo 2003 jan-fev; 13 (1): 20-45. 
64. Chaudhry SI, Krumholz HM, Foody JM. Systolic hypertension in older adults. JAMA 2004 Sep 1; 292 (9): 1074-80.

65. MacWalter R, Watt G, Cotton P. Hypertension in older people. A National Clinical Guideline. Edinburgh: Scottish Intercollegiate Guidelines Network; 2001.

66. Pearson TA, Blair SN, Daniels SR, Eckel RH, Fair JM, Fortmann SP, et al. AHA Guidelines for Primary Prevention of Cardiovascular Disease and Stroke: 2002 update: Consensus panel guide for comprehensive risk reduction for adult patients wi- thout coronary or other atherosclerotic vascular diseases. Circulation 2002 Jul 16; 106 (3): 388-91.

67. De Backer G, Ambrosioni E, BorchJonhsen K, Brotons C, Cifkova R, Dallongeville J, et al. European guidelines on cardiovascular disease prevention in clinical practice: Third Joint Task Force of European and other Societies on Cardiovascular Disease Prevention in Clinical Practise. Eur J Cardiovasc Prev Rehabil 2003 Aug; 10 (4): S1-S78.

\section{ABSTRACT}

Introduction: Several international organisms have been suggesting aerobic exercise as a strategy of prevention, treatment and control of high blood pressure in population in general. However, the effect of the aerobic exercise on the blood pressure of the elderly is still a barely studied area and the results of the surveys are not always steady.

Aim: This review aims to analyse the chronic and acute effects of the aerobic exercise on blood pressure levels of the aged population.

Method: A research was carried out through Pubmed and Highwire Press data base. There were also considered the recommendations of several international organizations in the area of cardiology. 67 articles were included in the study.

Conclusions: The aerobic exercise, either acute or chronic, seems to reduce the levels of both dinical and ambulatory blood pressures on aged individuals, especially the hypertensive ones. Therefore, it appears to subsist enough evidences to consider the aerobic exercise as a non-pharmaceutical therapeutic strategy, and as a change in the lifestyle, vital for the prevention, treatment and control of high blood pressure in aged individuals. Health professionals must have an active role in the recommendation of exercise to the elderly population, leading to the prevention and control of cardiovascular diseases.

Key-Words: Exercise; Blood Pressure; Aged. 